\title{
The Research and Design of Car Door Control System Based on CAN-Bus
}

\author{
HU Hao ${ }^{1}$, CHEN Yong-li ${ }^{1}$, YAN Ying-min ${ }^{1}$ \\ (1. Electrical engineering, Mechanical Engineering College. Shi jia zhuang 050003)
}

\begin{abstract}
Based on the purposes of the fast control of vehicle. CAN bus principle was used to design a personalized car control system method and Applicated CANoe software to design a four node control system, network node was definited, a message database was created, virtual panel and CAPL program was editde, the driver's operation was simulated as well as the operating system operation process. Drawn the conclusion of bus load rate and send the vehicle information to display system through the bus.
\end{abstract}

KeyWords: CAN technology, car door control, CAN protocol, J1939

\section{Fundamentals of control systems}

In accordance with the door control system structure and control requirements, the door control system according to the distributed modular thought formation control system configurations, each module are analyzed designed. The feature of the software is the ability to detect information errors frame during the processing of date frame and to report it to sender to initiate a new transmission[1]. Originally developed for the automotive industry, CAN is a fieldbus also suitable for general industrial applications[2].

\section{Door CAN bus simulation}

\subsection{CAN software system}

CANoe software is Vector Informatik de- veloped CAN tools series one of the most important tools .Vector Informatik is the German company specialized in a particular field bus CAN bus research, development of high-tech companies. It in CAN bus application fields and development of a series of powerful software development tool.CANoe ( CAN Open Environment ) as CAN bus development tools support the whole system development of the whole process.

CANoe software using Windows windows form to visually display the user interface, including for the bus design of Simulation Setup and Measurement Setup; for bus assessment of Trace, Write, Data, Graphics, Stastics and Bus Stastics. In addition, the CANoe also contains a three editor are: Panel Editor, $\mathrm{CANdb}++$ Editor and CAPL Browser[3,4]

\subsection{The network node definition and analysis}

This design only needs to construct the network physical layer and data link layer uses the ISO11898-1 standard to build, application layer identifier data format in accordance with SAE J1939 protocol format to set the communication rate of $250 \mathrm{Kbps}$

This design uses four nodes respectively: control node, sensor node, the display and realization. In the simulation of the CANoe configuration window, four nodes are respectively connected with red line to the bus, forming a four node net work simulation control system. Wherein each node corresponds to a panel, such as control nodes correspond to the control 
panel,sensor nodes correspond to sensor panel, display the node corresponding to the display panel, to achieve nodes correspond to achieve panel. Increase the intelligent node can expand the cover area of can bus, but following the increasingof intelligent node, the reliability of CAN Bus decreases[5].

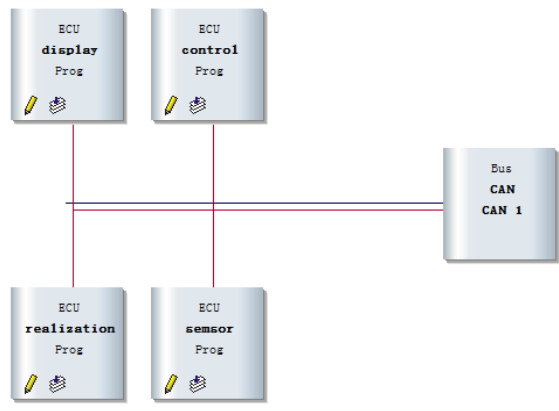

Fig. 1: Node connection

\subsection{Database establishment}

To establish a complete system to the design of their system has an overall grasp, to the control system should realize the function, control process of each node to send a message or to receive message should have a clear understanding. For signal definition includes signal name, length, byte order, signal value units, scope, type, scale factor, offset, in addition to the definition of the message signal and the receiving node. Message definition includes the message name, type, address, data byte length, contained in the signal and sending nodes and the receiving nodes. The environment variable is the control panel controls associated with the subscriber only channel. The controls will be the corresponding value of the environment variable is assigned to the message signal, and then sends a signal to the bus, through the bus transmission to the receiving node, the receiving node message signal is assigned to the control of the environment variables so that the control to achieve a predetermined action.

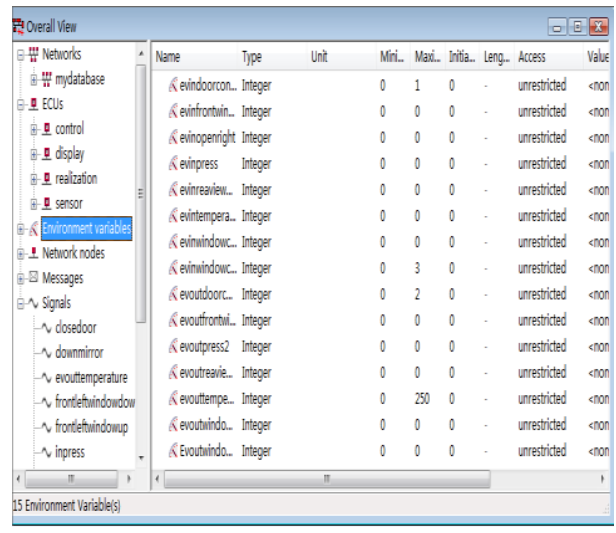

Fig. 2: Establishment of Database

The figure shows the database in mydatabase.dbc environment variable is defined. For example sensor input control panel corresponding environment variable ( including evinpress, evintemperature, evinwindow etc.), a control input panel corresponding environment variable ( including evindoorcontrol, evinwindowcontrol and so on ), output panel corresponding environment variable ( including evoutpress, evouttemperature, evoutwindow, etc. ) simulation panel corresponding environment variable ( including evoutdoorcontrol, evoutwindowcontrol and so on ) these environment variables defined effective guarantee for each node and the connection between the panels and communication, so in various interactive panel to complete the operation must be set before good node model, to establish a good database, ensure each control and corresponding environment variable contact, defined in the database messages and signals and program signal are corresponded, the system can achieve its functions.

\subsection{Panel Editor}

Panel editor panel control is mainly designed to environmental variables with the panel on the link, to realize virtual instrument system, which has the com- 
puter resources, interface circuit, user interface software such as the organic combination of function. The system introduced in this article the main editing four panel are respectively control the input panel, a sensor input panel and a vehicle information output panel with a virtual panel in the control input panel, is mainly a driver input some commands, such as door lock control and window control, in imitation of real is the panel main editor window position, mirror position and lock the vehicle control. Used to simulate actual components - reflect the driver input commands in the car component im- plementation. In the simulation process by controlling the input controls in the panel a signal is assigned to the environmental variables in way of signals transmitted through the bus to the simulation node through the CAPL program to signal assigned to the simulation of control panel of the control environment variables, which can make the simulation of the controls in the panel meets the control requirements, such as to control the car window, the state of the door lock And the angle of the rearview mirror and so on .

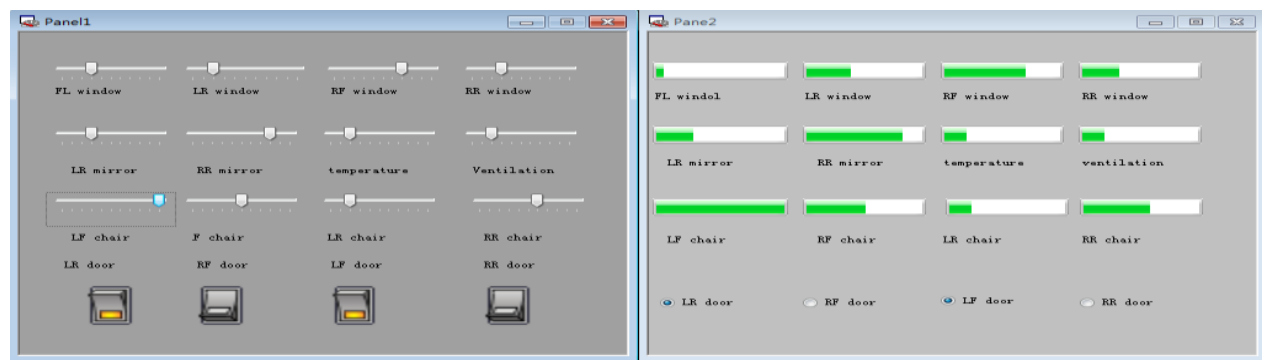

Fig. 3: Panel of Control and Implementation

In the sensor input panel, the main simulation of the sensor input signal. The car inside various the situation faithfully transmitted onto the bus. The real vehicle in such information is provided by the sensor measured and sent to the bus. In the integrated display system panel, using the direct display mode, that is to say the car information detected by the sensor in the display panel can be shown on the main interface design mainly includes: the interior pressure, the location of the window, the temperature inside the vehi- cle, residual fuel, driving speed, tire information, the remaining battery power. In the actual control of each instruction corresponds to a control action, control the movements of each is independent, such as the door or window control, as long as you press the button or pulling the slide bar can imagine the real parts of car is going to happen, each electric device corresponding to the car on the sensor or instrument is feasible in practice. Concrete panel design as shown in the figure:

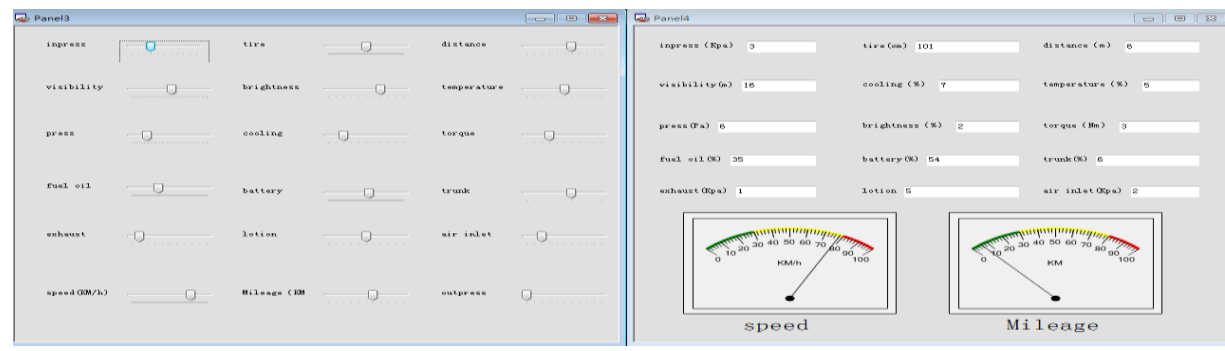

Fig. 4: Panel of Sensor Input and Display 


\section{Conclusion}

The simulation of the actual load rate from CANoe software bus Statistics (Bus Statics ) window that, used in the system bus is CAN1, so CAN2 bus the number 0 bus 1 , actual load rate is $35.22 \%$. Data transmission using extended frame, 700 frame per second send, so far has been transmitted 19872 frames. Send data frames per second can be estimated through information sending and receiving cycle per second, which sends data frame number is 710 frames. In addition to the design of baud rate is $250 \mathrm{Kbps}$.

Bus message types can only be a data frame, there is no error frame, remote frame and overload frames. Among them, start of frame 1, arbitration domain 29 ( extended frame format), the control domain has 6, 64 bit data domain, CRC domain 15 , answering domain 2 , end of frame 7 , a total of 124 .

The above data into the formula, bus load rate $=\left(\begin{array}{lll}124 & \mathrm{x} & 710\end{array}\right) /$ $250000=35.216 \%$.

\begin{tabular}{l|ll}
\hline & CAN 1 & CAN 2 \\
\hline Busload [\$] & 35.22 & 0.00 \\
Peakload [\$] & 35.22 & 0.00 \\
Std. Data [fr/s] & 0 & 0 \\
Std. Data [total] & 0 & 0 \\
Ext. Data [fr/s] & 710 & 0 \\
Ext. Data [total] & 19872 & 0 \\
Std. Remote [fr/s] & 0 & 0 \\
Std. Remote [total] & 0 & 0 \\
Ext. Remote [fr/s] & 0 & 0 \\
Ext. Remote [total] & 0 & 0 \\
Errorframe [fr/s] & 0 & 0 \\
Errorframes [total] & 0 & 0 \\
Chip state Active & Active
\end{tabular}

Fig.5 : Bus Statistics

The error between oretical load rate and the actual load rate is: $\frac{35.22 \%-35.216 \%}{35.22 \%} \times 100 \%=0.0114 \%$

\section{Reference}

[1] Zhai Li, Gu Zhongli Zhang. Chengning, Guo Fen, Sun Fengchun.ociety. A New Communication Method Based on PLC and CAN BUS for Electric Vehicle[J]. The Seventh International Conference on Electronic Measurement and Instruments, 2005, 4: 167-169.

[2] Fuhua Kuang, Bugong Xu. An Implementation of CANopen at Water Eletrolysis Hydrogen Generation Station. 2010 ISECS International Colloquium on Computing, Communication, Control, and Management(CCCM), 2010, pp.738-740

[3] E.Jmanders, L.A.Barford, and G.Biswas,"An approach for fault detection and isolation in dynamic systems from distributed measurements," IEEE Transactons on instrumentation and Measurement, vol.51, No.2, 2002.

[4] M.Ellims, S. Parker, and J. Zurlo, "Design and analysis of a robust real-time engine control network ," IEEE Micro, vol. 22, No.4,2002.

[5] WANG Feng An emulation design and analysis of vehicle integrated information display system based on J1939 protocol[J], Modern Computer, 2007, 5(1) :20-30. 